\title{
Quantification de l'ablation d'un bassin versant marno-calcaire alpin durant le Petit Age Glaciaire par l'étude d'un système lacustre (cas du lac du «Claps » de Luc-en-Diois Drôme, France)
}

\author{
Jean-François Buoncristiani ${ }^{\mathrm{a}, *}$, Christophe Petit ${ }^{\mathrm{a}}$, Michel Campy ${ }^{\mathrm{a}}$, Gilles Bossuet ${ }^{\mathrm{b}}$, \\ Hervé Richard ${ }^{\mathrm{b}}$
}

${ }^{a}$ Centre des Sciences de la Terre, UMR 5561, 6 Bd Gabriel, 21000 Dijon, France

${ }^{\mathrm{b}}$ CNRS, UMR 6565 Chrono-Ecologie, UFR Sciences et Techniques, 16 route de Gray, 25030 Besançon, France

\begin{abstract}
Résumé
Les mesures de l'érosion actuelle donnent des résultats hétérogènes en fonction des méthodes utilisées. Cet article propose une approche de l'érosion durant le Petit Age Glaciaire par l'analyse quantitative des matériaux détritiques piégés dans un lac de barrage. Ce remplissage est reconnu par deux forages et ces observations ponctuelles sont complétées par une étude en sismique réflexion fournissant une estimation fiable de la géométrie du remplissage lacustre. La production sédimentaire est calculée à partir des trois données nécessaires : (1) la surface du bassin producteur, (2) la durée de fonctionnement du système et (3) le volume de sédiments piégés. A partir des trois variables déterminées, nous proposons un taux de production sédimentaire détritique de $1846 \pm 145$ tonnes $/ \mathrm{km}^{2} / \mathrm{an}$, ce qui représente une dénudation moyenne de 0,68 $\pm 0,05 \mathrm{~mm} / \mathrm{an}$. L'importance d'un forçage hydro-climatique et anthropique sur la production sédimentaire correspondante au cours du Petit Age Glaciaire est soulignée par cette étude.
\end{abstract}

\begin{abstract}
Measurements of present-day erosion may give heterogeneous results according to the methods used. This article proposes an approach to erosion during the Little Ice Age by the quantitative analysis of detritical materials trapped in a dammed lake. This infill is recognized through two drillings, and these specific observations are supplemented by a seismic reflexion survey which provided a reliable estimate of the geometry of the lake infill. The sedimentary production is calculated from three pieces of data: (1) the surface of the drainage area, (2) duration of the lake system activity and (3) the volume of trapped sediments. From the three determined variables, we propose a detritical production sedimentary rate of $1846 \pm 145$ tonnes $/ \mathrm{km}^{2} /$ year, which represents a mean denudation of $0.68 \pm 0.05 \mathrm{~mm} / \mathrm{year}$. The importance of a hydro-climatic and anthropic forcing on the sedimentary production during the Little Ice Age is emphasized by this study.

Éditions scientifiques et médicales Elsevier SAS. All rights reserved.
\end{abstract}

Mots clés: Production sédimentaire; Dénudation; Sismique réflexion; Climat; Petit Age Glaciaire

Keywords: Sediment yield; Denudation; Seismic reflection; Climate; Little Ice Age

\section{Introduction}

L'étude quantitative de la production sédimentaire spécifique (en tonnes $/ \mathrm{km}^{2} / \mathrm{an}$ ) est abordée par différentes méthodes : soit par l'analyse de petits bassins-versants expéri-

\footnotetext{
* Corresponding author.

E-mail address: jean-francois.buoncristiani@u-bourgogne.fr (J.F. Buoncristiani).
}

mentaux [1,2] ou de parcelles 3,4, soit par la mesure des produits exportés à l'embouchure des rivières [5,6] Les résultats obtenus montrent que les valeurs de la production sédimentaire sont comprises entre $10 \mathrm{t} / \mathrm{km}^{2} / \mathrm{an}$ pour les fleuves drainant des zones de boucliers de faible altitude (boucliers canadien et sibérien) et plus de $10000 \mathrm{t} / \mathrm{km}^{2} / \mathrm{an}$ pour les régions montagneuses de l'Himalaya et des îles du Pacifique. Ces données nous renseignent bien sur le fonctionnement des systèmes actuels, cependant elles ne permettent pas une extrapolation sur de longues périodes, du fait de 
leur faible représentativité dans le temps et l'espace. La grande amplitude de ces valeurs est due à la variabilité des conditions locales et en particulier à trois paramètres principaux que sont la lithologie du substrat, le relief (valeur des pentes ou « rugosité ») et le climat.

Pour différencier l'influence de ces trois paramètres sur l'intensité des processus d'érosion, un certain nombre de travaux ont été réalisés sur des plus petits bassins versants présentant une homogénéité vis-à-vis de ces trois critères [7.8.9]. La production sédimentaire due à l'érosion peut être déduite de l'étude d'un piège sédimentaire continental, représenté par un géosystème lacustre, que l'on peut décomposer en deux systèmes : le bassin producteur (le bassin versant) et le bassin récepteur (le remplissage lacustre). Ainsi en quantifiant les différentes variables de ce géosystème, la durée de son fonctionnement, la surface de son bassin producteur et le volume de sédiments piégés, on pourra alors calculer la production sédimentaire moyenne de son bassin versant et son taux d'érosion.

En prenant comme exemple un remplissage lacustre alpin : le paléo-lac du «Claps» de Luc-en-Diois (Drôme, France), nous proposons de quantifier le volume de sédiment piégé par le système récepteur afin de calculer la production sédimentaire détritique. Ce lac a été choisi car sa durée de fonctionnement, qui est parfaitement connue, correspond à la dernière dégradation climatique (le Petit Age Glaciaire); de plus le remplissage sédimentaire est particulièrement dilaté. Il présente un double intérêt, permettant de discuter ainsi le rôle des contraintes hydroclimatiques et lithologiques sur l'érosion du bassin-versant de ce lac historique.

\section{Le système limnologique du «Claps » de Luc-en- Diois}

\subsection{Le bassin producteur}

Le cours amont de la Drôme (Fig. 1a), a été barré par l'effondrement d'un épais banc de calcaire (le «Claps », Fig. 3) dont le volume est voisin d'un million de mètres cube. Du fait de la topographie initiale cet écroulement s'est divisé en deux lobes, ce qui a permis la formation de deux lacs, un grand lac amont et un petit lac aval (Fig.2). Située à une altitude moyenne de $500 \mathrm{~m}$, la Drôme présente dans ce secteur un régime hydro-climatique de type préalpin caractérisé par des crues violentes intervenant au printemps et à l'automne 10.11.

Le «Claps» correspond à un effondrement de la barre calcaire tithonique redressée du flanc nord du synclinal de Lesches-Beaumont et le grand lac occupe la large vallée installée dans les marnes du Crétacé inférieur (Fig. 1b). L'analyse de la topographie permet de délimiter le bassin versant du lac ; sa surface calculée par analyse d'image est

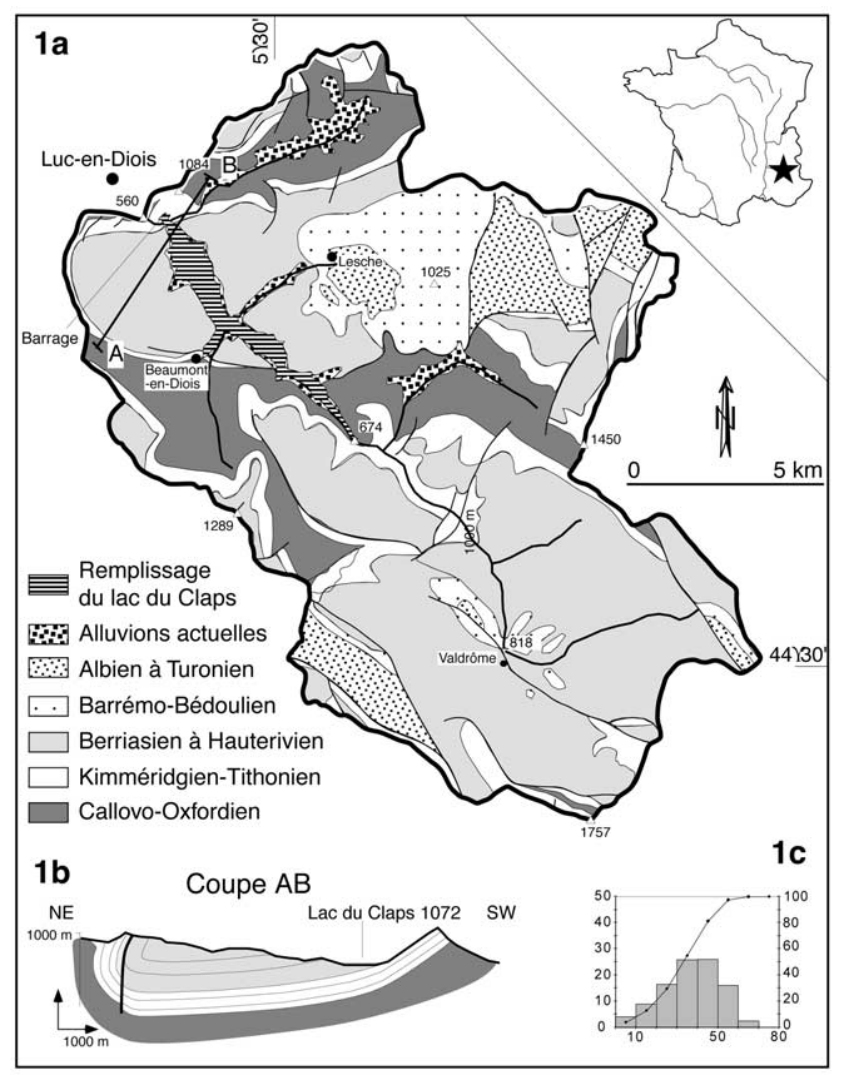

Fig. 1.1a : Situation de la zone d'étude : le lac du «Claps » de Luc-en-Diois (Alpes, Drôme), et géologie du bassin versant producteur (tirée de la carte géologique Luc-en-Diois 1/50000). $1 \mathrm{~b}$ : coupe géologique simplifiée recoupant le synclinal de Lesches-Beaumont et le grand lac installé dans les marnes du Crétacé inférieur. 1c : Histogramme des pentes du bassin versant.

1a : Situation of the studied area: Luc-en-Diois "Claps" lake (Alps, Drôme), and geology of the watershed (drawn from the geological map Luc-en-Diois 1/50000). 1b: simplified section across the LeschesBeaumont syncline and the large lake developed on early Cretaceous marls. $1 \mathrm{c}$ : Histogram of the slopes of the watershed.

de $187 \mathrm{~km}^{2}$ avec une incertitude de $3 \%$ liée à la présence de la barre de calcaires karstifiés qui délimite fréquemment le bassin versant (Fig. 1).

Les roches du bassin versant peuvent être regroupées en fonction de leur résistance à l'érosion mécanique: des calcaires $(23 \%)$ qui forment les falaises (Fig. 3), et des marnes et marno-calcaires (77\%) qui déterminent des versants moins pentus affectés fréquemment par des «bad lands ». Le relief du bassin versant est analysé d'après ces pentes qui sont calculées à partir d'un modèle numérique d'altitude (pas de $100 \mathrm{~m}$ ). Elles s'échelonnent entre $5^{\circ}$ et $80^{\circ}$. Les pentes les plus fortes correspondent aux falaises calcaires bordant le bassin versant. Les valeurs moyennes de pente sont fortement représentées dans ce bassin versant et correspondent aux zones marno-calcaires et aux zones d'éboulis. Puis les faibles valeurs de pentes représentent les zones tabulaires calcaires et alluvionnaires (remplissage lacustre). 


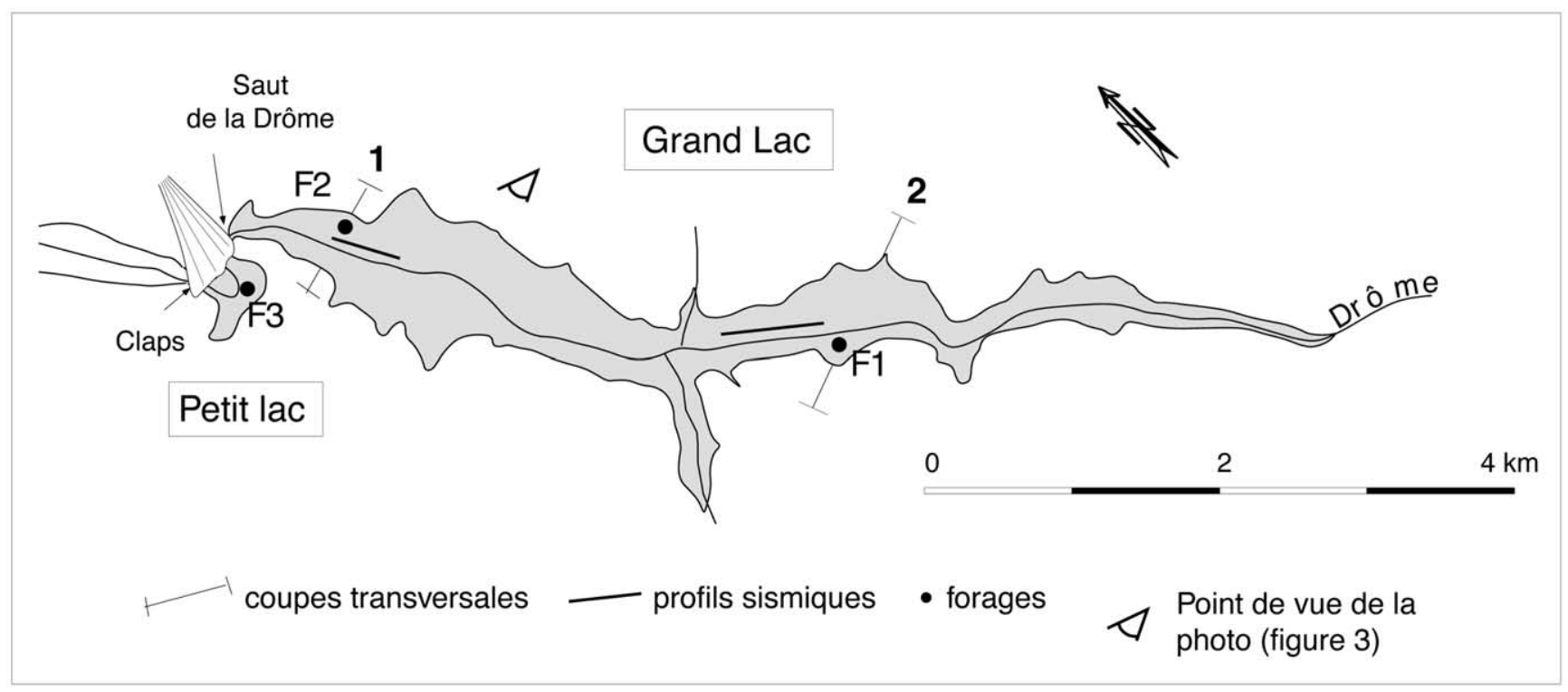

Fig. 2. Limites du remplissage lacustre et emplacement des coupes transversales, des forages, et des profils sismiques.

Limits of the lake fill and location of transversal section, boreholes, and seismic profiles.

\subsection{Durée de fonctionnement du système lacustre} et caractéristique sédimentologique du remplissage

Actuellement, le remplissage sédimentaire du lac occupe la totalité de la vacuité initialement disponible (Fig. 3). D'après les données d'archives, l'écroulement du banc calcaire est daté entre 1442-1447 [12], et l'assèchement artificiel du lac résiduel a été réalisé par des moines entre 1790-1795 en perçant une conduite dans le barrage. La durée de fonctionnement du piège sédimentaire est donc estimée à 348 ans \pm 10 ans. Le remplissage lacustre enregistre presque la totalité de la crise climatique du Petit Age

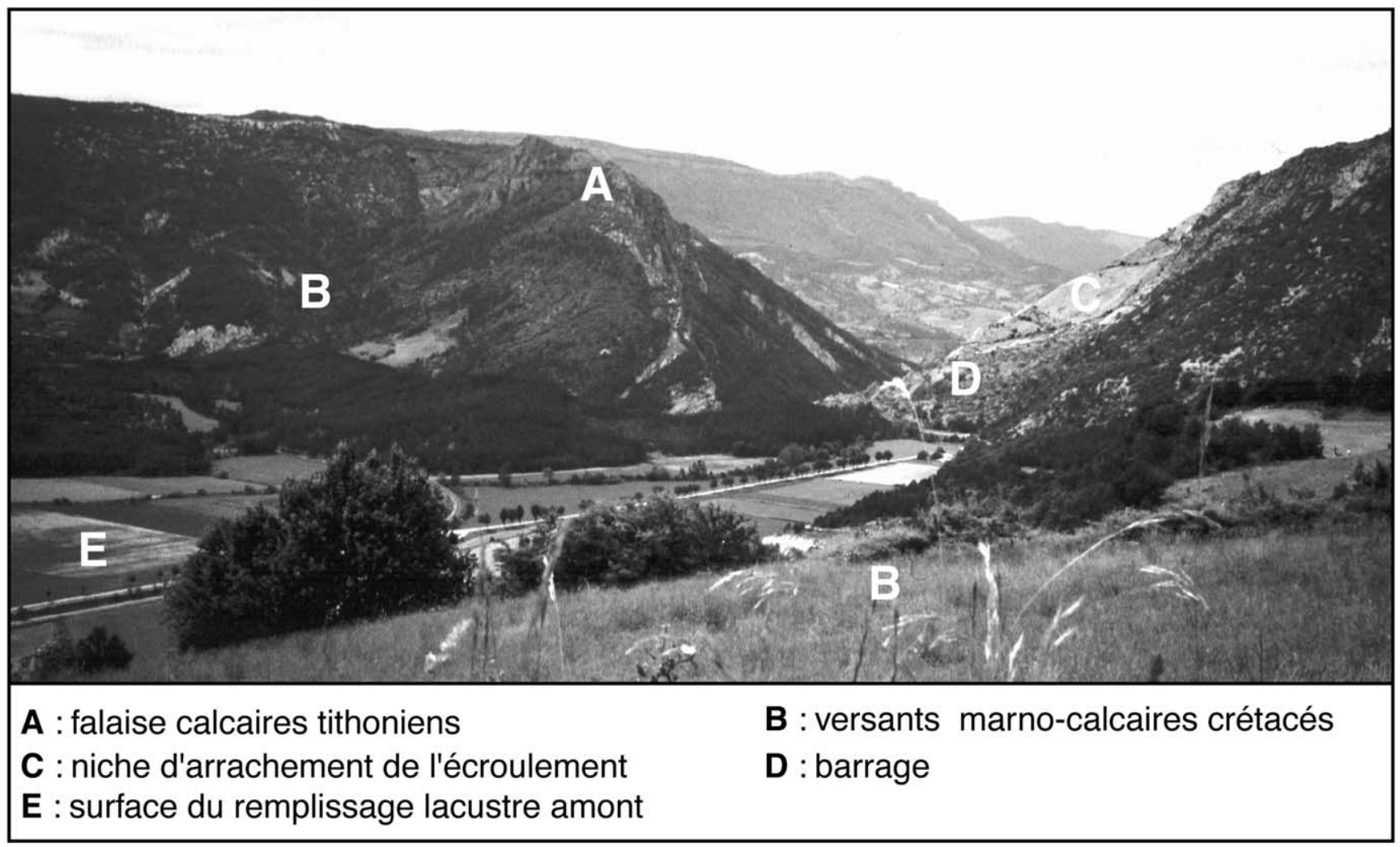

Fig. 3. Vue de la surface du remplissage lacustre (E), de la zone d'éboulement (C-D) et de la morphologie des versants (A-B). View from the surface of lake infill (E), the zone of crumbling (C-D), and the morphology of slopes (A-B). 
Glaciaire défini dans les Alpes entre 1550-1850 et caractérisé par une baisse des températures de 0,5 à $1{ }^{\circ} \mathrm{C}$ et par des enneigements plus tardifs par rapport à l'actuel [13,14].

Deux forages à but géotechnique et non carottés ont été réalisés dans le remplissage du grand lac. Ils permettent cependant l'identification des principaux faciès du remplissage lacustre. Un forage amont (F1, Fig. 2), situé en bordure de la vallée montre $30 \mathrm{~m}$ d'une formation essentiellement sablo-graveleuse avec quelques passées argileuses. Le forage aval (F2, Fig. 2) a atteint le substrat calcaire à $65 \mathrm{~m}$. Il se décompose de la façon suivante : $3 \mathrm{~m}$ de sables graveleux à la base, interprétés comme les alluvions de la Drôme avant l'effondrement, puis $63 \mathrm{~m}$ de remplissage d'argiles silteuses. Ces deux forages permettent d'illustrer, au sein du remplissage du lac amont, un gradient granulométrique entre un pôle proximal grossier et un pôle distal fin.

Un forage carotté (F3, Fig. 2) réalisé dans le petit lac a recoupé les six premiers mètres du remplissage. La partie supérieure $(0$ à $2,5 \mathrm{~m})$ correspond à des silts argileux bioturbés jaunes; l'assèchement du lac a permis le développement d'un sol hydromorphe sous une prairie humide. Les carottes plus profondes (2,5 à $6 \mathrm{~m})$ montrent une succession de sédiments rythmés d'épaisseur centimétrique. Les séquences élémentaires qui débutent par une surface basale bien marquée, parfois érosive, sont constituées de silts grossiers argilo-sableux ( $15 \%$ de 0 à $2 \mu \mathrm{m}, 30 \%$ de 2 à $20 \mu \mathrm{m}, 45 \%$ de 20 à $50 \mu \mathrm{m}$ et $10 \%$ supérieur à $50 \mu \mathrm{m}$ ) passant à des silts argileux (20\% de 0 à $2 \mu \mathrm{m}, 50 \%$ de 2 à $20 \mu \mathrm{m}, 30 \%$ de 20 à $50 \mu \mathrm{m})$. Les observations au microscope n'ont pas permis l'identification de constituant biogé- nique dans ces séquences. De ce fait, nous considérons que ces séquences sont détritiques, issues du bassin versant et mises en place lors de crues élémentaires dans le petit lac alors que les sédiments plus grossiers ont pu être piégés en amont dans le grand lac.

\section{Géométrie du remplissage}

\subsection{Les données d'affleurement et de sismique réflexion}

À partir des altitudes du cours actuel, mesurées de part et d'autre du lac $(674 \mathrm{~m}$ en amont et $560 \mathrm{~m}$ en aval du «Claps ») nous avons déduit le profil en long de la Drôme antérieur à l'éboulement (Fig. 4). La pente moyenne de ce profil est estimée à $0,7 \%$. Deux coupes transversales construites d'après les données des forages et la topographie des versants de la vallée permettent de rendre compte de la morphologie générale du remplissage lacustre en considérant le mur des alluvions horizontal (Fig. 4).

Afin de déterminer de façon plus précise la géométrie de la surface anté-lacustre, nous avons effectué une investigation sismique réflexion du remplissage. Cette méthode a déjà largement été utilisée pour caractériser la nature et la géométrie des remplissages lacustres [15.16.17.18.19].

La technique employée dans cette étude est une acquisition en couverture multiple 20] Les mesures ont été réalisées avec un sismographe à 24 canaux et des géophones verticaux de $10 \mathrm{~Hz}$ comme récepteurs. La fenêtre d'acquisition est de $200 \mathrm{~ms}$ et un filtre passe-bas $50 \mathrm{~Hz}$ est utilisé à

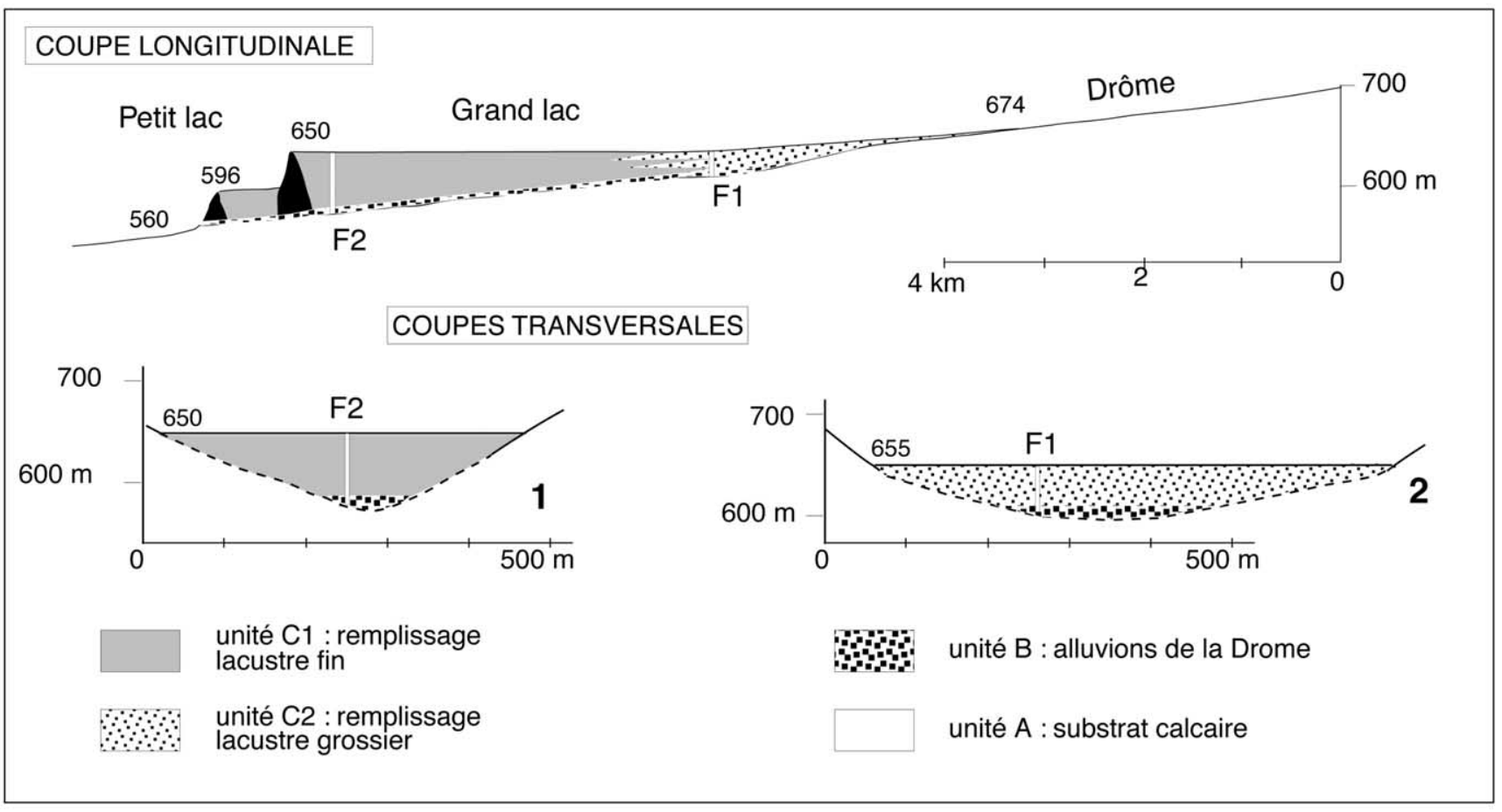

Fig. 4. Coupe longitudinale et coupes transversales dans le remplissage lacustre, réalisées à partir des forages mécaniques et des profils sismique réflexion. Longitudinal and transversal sections of the lacustrine infilling from bore hole data and seismic reflection profiles. 
l'acquisition afin de diminuer la saturation sur les basses fréquences 21$]$

L'enregistrement s'est fait en ligne avec un espacement entre les géophones et entre les tirs identiques de 5 mètres. La couverture réalisée par ce type de dispositif est d'ordre 12 et fournit des points miroirs (CMP) espacés de 2,5 m. La source d'onde utilisée est constituée par un choc entre une masse de $8 \mathrm{Kg}$ et une plaque de «dural » favorisant ainsi un signal hautes fréquences. Les tirs sont effectués le long d'une route compactée, ce qui permet un bon couplage avec le sous-sol, une bonne profondeur d'investigation, et un haut spectre de fréquence des signaux.

Le traitement inclut les étapes classiques [22]: statique sur réfraction par rapport à un plan de référence (premières arrivées à vitesse constante), contrôle des gains automatiques (grande fenêtre : $200 \mathrm{~ms}$ ), filtre passe-bande trapézoïdale $80-120 \mathrm{~Hz} / 250-300 \mathrm{~Hz}$, effacement des bruits non encore filtrés (ondes de surface, bruit du tir dans l'air), analyse de vitesse, correction de la distance de la source aux géophones, statique résiduelle (fenêtre de $80 \mathrm{~ms}$ ), sommation des traces en points miroirs (CMP), déconvolution en phase nulle.

Après traitement, les profils sismiques obtenus présentent une fréquence moyenne comprise entre $150-200 \mathrm{~Hz}$, on peut ainsi estimer que la résolution théorique verticale représente $5 \mathrm{~m}$ (1/4 de la longueur d'onde [22] $)$. La conversion temps/profondeur est effectuée grâce à un modèle de vitesse déterminé d'après les enregistrements réfraction et les vitesses de correction dynamique.

Afin de s'affranchir des effets de bords latéraux, deux profils sismiques longitudinaux ont été effectués. L'un dans la partie amont du remplissage lacustre, et l'autre dans la partie aval (Fig. 2). Sur ces profils, les mêmes sismofaciès sont retrouvés. Le profil amont présente trois sismofaciès différents, décomposés de la façon suivante de la base au sommet (Fig. 5) :

- unité $\mathrm{A}$ (basale) : le toit de cette unité $(85 \mathrm{~ms}$ à $90 \mathrm{~ms})$ montre des réflexions de forte amplitude sub-parallèles, et une vitesse très rapide $(3250 \mathrm{~m} / \mathrm{s}$ d'après la réfraction) ;

- unité B (médiane) : elle présente des réflecteurs discontinus (entre $80 \mathrm{~ms}$ à $50 \mathrm{~ms}$ ) et de faible amplitude de réflexion ;

- unité C (sommitale) : sur cette unité (à partir de $50 \mathrm{~ms}$ ) pas ou peu de réflexion sont visibles; elle se caractérise par une zone transparente de faible vitesse $(1000 \mathrm{~m} / \mathrm{s}$ sur le profil amont et $1200 \mathrm{~m} / \mathrm{s}$ sur le profil aval d'après la réfraction).

À partir des résultats des forages du remplissage, il est possible de mettre en relation les sismofaciès individualisés dans la section sismique et les différentes unités géologiques (Fig. 4). L'unité A basale de forte amplitude et dont la vitesse est rapide correspond au substrat de calcaires tithoniens. L'unité B qui représente sur les deux sections une

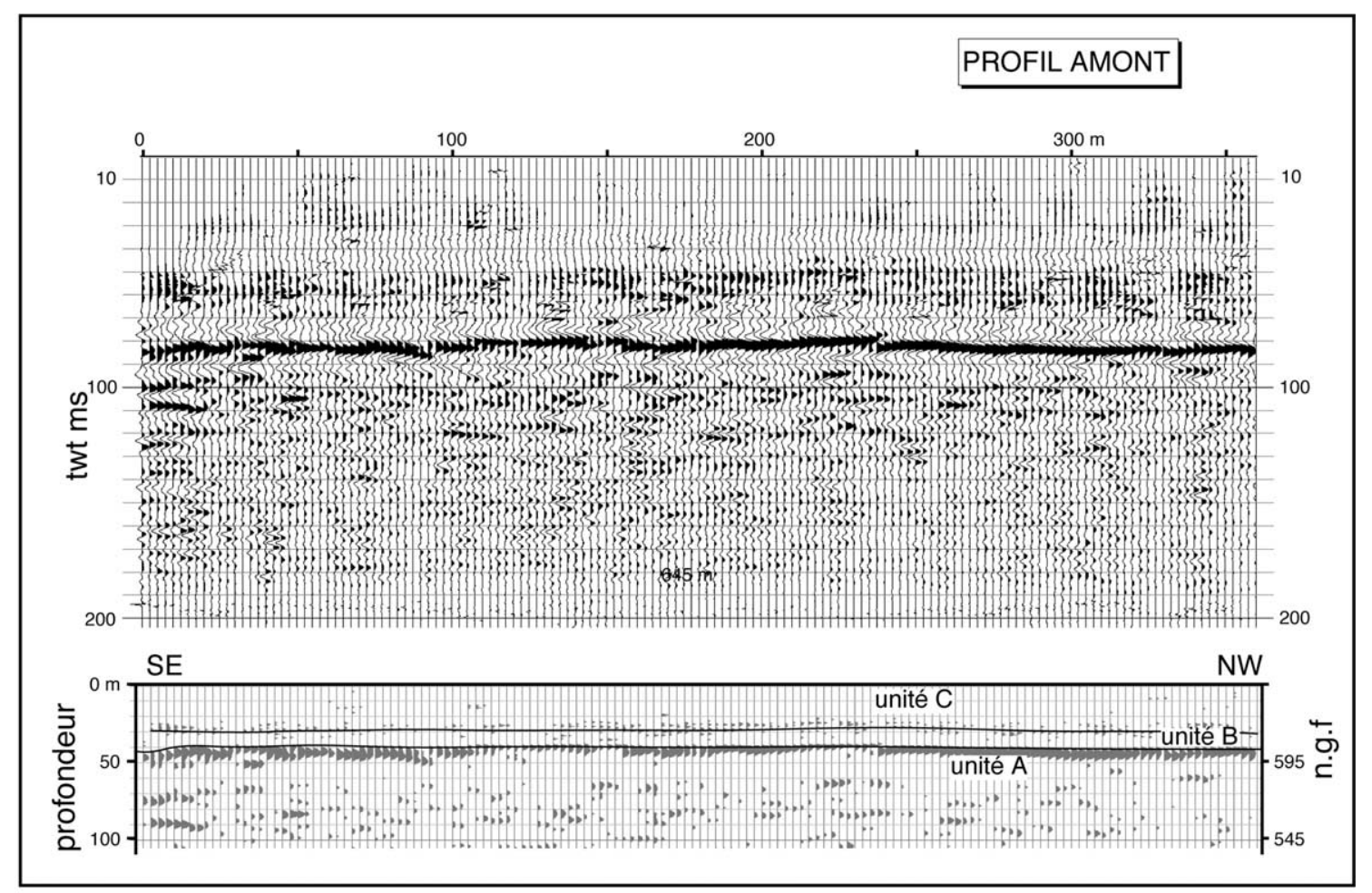

Fig. 5. Profil sismique réflexion amont après traitement en miliseconde temps-double - profil sismique en coupe profondeur (en mètre) à partir du modèle de vitesse et interprétation des différents sismofaciès.

Upstream seismic reflection profile (F.Drome) after processing, two way travel time in ms, seismic profile depth (m) according to the velocity model and interpretation of the different units. 
épaisseur moyenne de $10 \mathrm{~m}$ est interprétée comme étant formée par les alluvions de la Drôme avant l'effondrement. L'unité $\mathrm{C}$ de faible vitesse correspond aux sédiments fins du remplissage lacustre. Les variations de lithologie de l'amont à l'aval se marquent dans les vitesses de ces formations sur les deux profils. L'unité C présente une vitesse de $1000 \mathrm{~m} / \mathrm{s}$ dans le profil amont, ce qui correspond à des sédiments sablo-argileux, alors que sur le profil aval, cette même unité est caractérisée par une vitesse de $1200 \mathrm{~m} / \mathrm{s}$, ce qui représente des sédiments plutôt argileux [23]

\subsection{Reconstruction de la surface inférieure du lac et volume stocké :}

La paléo-surface est reconstruite à partir des données de forages, d'affleurement, du profil en long et des profils sismiques, soit environ une cinquantaine de points. La méthode d'interpolation choisie est le krigeage, car cette technique utilise les variables régionalisées. Elle nécessite au préalable une analyse géostatistique des données et aboutit à un algorithme d'interpolation fonction de la variabilité statistique des données de base, unique pour chacun des cas d'études [24]. Afin d'éviter au maximum les problèmes liés aux effets de bords durant l'interpolation, nous avons ajouté comme données les versants de la vallée actuelle en se basant sur les cartes topographiques

Nous avons obtenu un modèle de la surface inférieure du remplissage lacustre (Fig. 6). La reconstruction de la paléosurface indique l'existence d'une vallée relativement encaissée (entre $590 \mathrm{~m}$ à l'aval et $680 \mathrm{~m}$ à l'amont), avec des versants de pente forte (entre 7 et $10^{\circ}$ ) sur cette portion du cours de la Drôme, qui représente cependant la continuité des versants adjacents au lac.

Ayant défini la surface inférieure du remplissage et connaissant sa surface supérieure, nous pouvons déterminer le volume de sédiments piégés dans le lac durant son fonctionnement car il correspond à l'intersection de ces deux surfaces. Il est calculé par intégrale (méthode de Simpson) et représente $70 \cdot 10^{6} \mathrm{~m}^{3}$ avec une incertitude de $10 \%$ (soit $\pm 3,510^{6} \mathrm{~m}^{3}$ ) estimée d'après l'erreur commise sur la reconstitution des surfaces [25]

\section{Production sédimentaire moyenne et dénudation du géosystème}

Connaissant le volume de sédiments stockés $\left(70,10^{6} \mathrm{~m}^{3}\right)$ et la durée de fonctionnement ( $348 \pm 10$ ans) du système, le taux de stockage moyen (Qe) en $\mathrm{m}^{3} /$ an est calculé :

$Q_{e}=V / t \quad \mathrm{~V}:$ Volume de sédiment stocké $\left(\mathrm{en}^{3}\right)$ t: durée de fonctionnement du système (en années ).
Il représente $0,2 \pm 0,0110^{6} \mathrm{~m}^{3} / \mathrm{an}$. Connaissant la surface du bassin récepteur $\left(\mathrm{S}_{\mathrm{r}}=1,610^{6} \mathrm{~m}^{2}\right)$ nous pouvons calculer un taux de sédimentation moyen dans le lac $(\mathrm{Qe} / \mathrm{Sr})$ qui représente : $12 \mathrm{~cm} / \mathrm{an}$.

La densité mesurée sur les carottes pour ces sédiments est de 1,8 tonnes $/ \mathrm{m}^{3}$, elle permet d'exprimer ce taux de stockage moyen $(\mathrm{Qe})$ en masse ( $\mathrm{Qm}$ en tonnes/an) :

$$
\begin{array}{r}
Q_{m}=Q_{e} X d \mathrm{Q}_{\mathrm{e}}: \text { le taux de stockage moyen }\left(\mathrm{en}^{3} / \mathrm{an}\right) \\
\mathrm{d} \text { : la masse volumique (en tonnes/an ). }
\end{array}
$$

Nous obtenons ainsi, une masse de sédiments piégés équivalente à $0,3 \pm 0,0210^{6}$ tonnes/an.

Pour estimer la production sédimentaire spécifique du bassin versant, il est nécessaire de déterminer l'efficacité du piégeage lacustre. Des travaux réalisés sur différents lacs montrent l'existence d'une relation logarithmique entre l'efficacité du piégeage et le temps de séjour des eaux dans le lac (Fig. 7) [26.27]. Il a été montré que cette relation s'applique à la fois pour un épisode hydrologique isolé (crue), et pour une approche à un pas plus grand en fonction d'une moyenne de débit [28]. En se basant sur cette méthode, il est nécessaire de connaître le temps de séjour des eaux dans le lac. Pour son calcul, nous allons considérer que la majeure partie des eaux viennent du cours de la Drôme. Pour cette rivière, fonctionnant durant le Petit Age Glaciaire, le régime hydro-climatique était alors différent. Mais, ne connaissant pas ce régime, nous allons baser notre calcul sur les données récentes :soit un débit moyen annuel de $3 \mathrm{~m}^{3} / \mathrm{s}$ (sur 50 ans) et un débit instantané de crue de $60 \mathrm{~m}^{3} / \mathrm{s}$ (données DIREN Rhône-Alpes). Ces valeurs fournissent respectivement des temps de séjour de 0,037 et 0,74 année. Puis, en reportant ces valeurs sur la courbe de Brune (Fig. 7) la capacité de piégeage du lac est estimée, elle varie entre 72 et $97 \%$. Cette fourchette de valeur étant relativement importante et résultant d'un certain nombre de simplifications (paléo-débit, alimentation en eau, nature du barrage), le volume de sédiment piégé dans le lac ne sera pas corrigé. Nous considérerons que la production sédimentaire détritique du bassin versant calculée représente une valeur minimum.

Fonction de la surface du bassin versant $\left(187 \pm 3 \mathrm{~km}^{2}\right)$, la production sédimentaire spécifique $(\mathrm{Qs})$ en tonnes $/ \mathrm{km}^{2} / \mathrm{an}$ s'exprime par :

$$
Q_{s}=Q_{m} / S_{e} \mathrm{Q}_{\mathrm{m}}=\text { le taux de stockage moyen (en tonnes/an) }
$$

Se: la surface du bassin producteur ( en $\mathrm{km}^{2}$ )

Elle est donc équivalente à $1846 \pm 145$ tonnes $/ \mathrm{km}^{2} / \mathrm{an}$. En considérant que le bassin versant est homogène, et constitué essentiellement de roches carbonatées, la production sédimentaire peut être convertie en dénudation à partir de la masse volumique apparente de $2,7 \mathrm{t} / \mathrm{m}^{3}$ [29]. On obtient ainsi une valeur de : 0,68 $\pm 0,05 \mathrm{~mm} / \mathrm{an}$. 


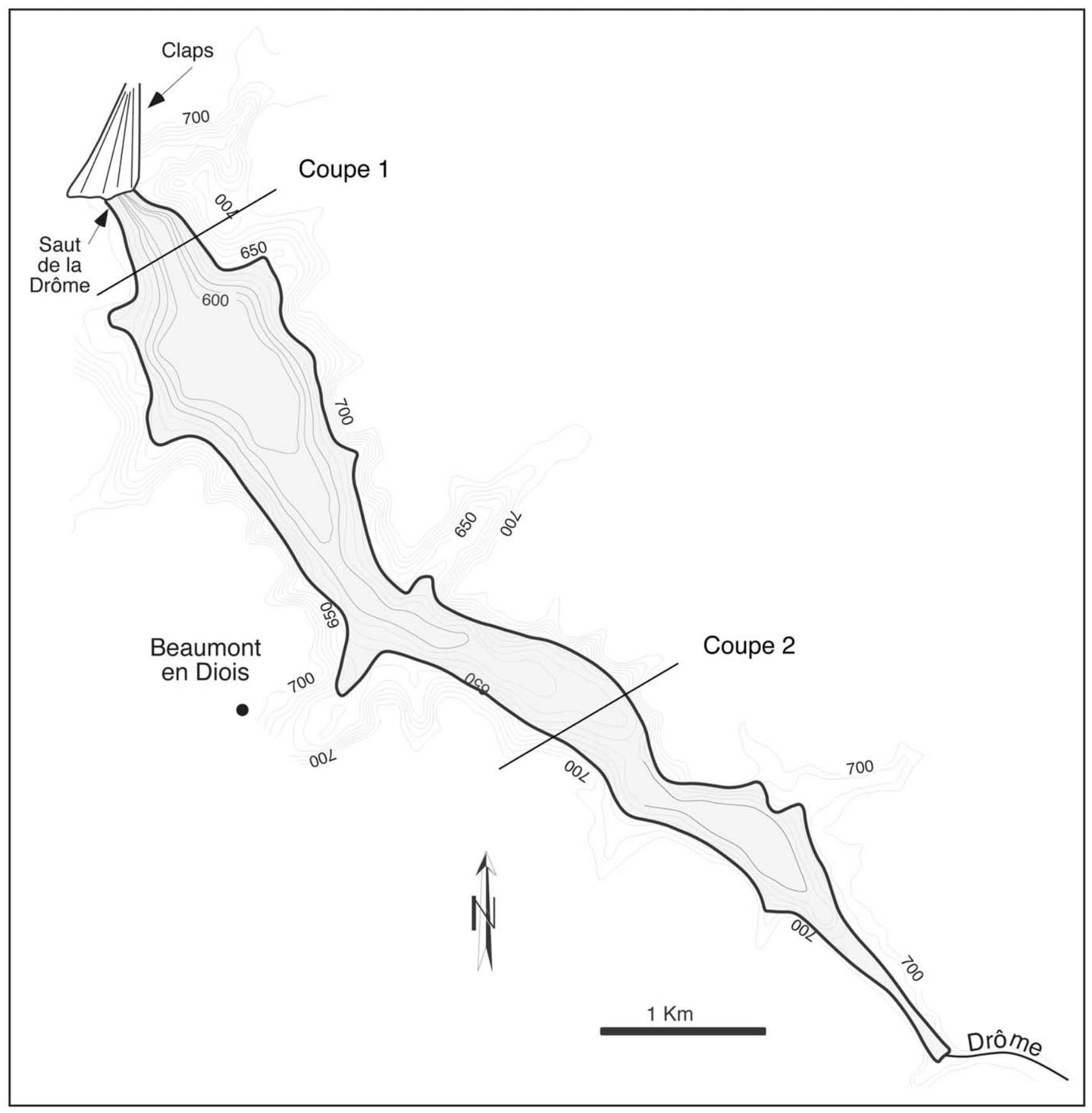

Fig. 6. Modélisation de la paléosurface correspondant à la base du remplissage lacustre en intégrant les versants, et limite du remplissage lacustre. Modeling of the paleosurface corresponding to the base of the lake infill integrating the slopes, and limit of the lake infill.

\section{Discussion et conclusion}

L'approche intégrée des différentes variables du géosystème lacustre du «Claps » de Luc-en-Diois a permis d'estimer à $1846 \pm 145$ tonnes $/ \mathrm{km}^{2} / \mathrm{an}$ la production sédimentaire spécifique moyenne pour une durée voisine de 350 ans (1442-1790). Ce système nous permet d'aborder l'influence d'une part de la lithologie (facteur passif) et d'autre part du climat et de l'anthropisation (facteurs actifs) sur l'intensité de l'érosion.
Les études menées sur des bassins-versants monolithologiques montrent que l'intensité de l'érosion mécanique actuelle couvre trois ordres de grandeur en fonction de la nature des roches [28]. Cependant, deux exemples fournissent des valeurs proches de celles calculées pour le bassin versant du « Claps » de Luc-en-Diois. Des travaux menés en Afrique du Nord montrent que des bassins producteurs de type «bad-land» ont des valeurs s'échelonnant entre 1000 et $2000 \mathrm{t} / \mathrm{km}^{2} / \mathrm{an}$ [30] Pour le fleuve Jaune (Huang Ho) dont le bassin versant est constitué par des lœss, les valeurs 


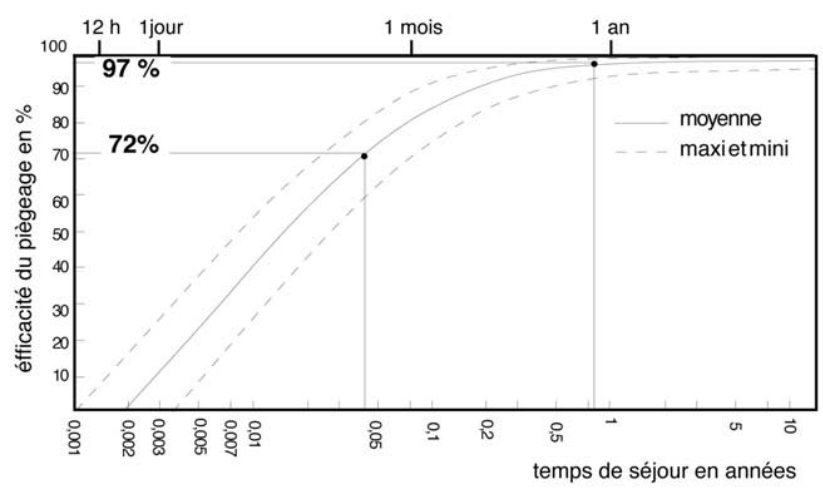

Fig. 7. Relations entre l'efficacité du piégeage sédimentaire et le temps de séjour dans le lac (modifiée de Brune, 1953), permettant de calculer l'efficacité du piégeage pour le lac du «Claps » de Luc-en-Diois.

Relations between the efficacity of the sedimentary trapping and the residence time in the lake (modified after Brune, allowing to calculate the efficacity of the sedimentary trapping for Luc-en-Diois "Claps" lake.

mesurées pour les 40 dernières années indiquent une production sédimentaire spécifique de $1400 \mathrm{t} / \mathrm{km}^{2} / \mathrm{an}$ [3]

Dans les Alpes, les mesures actuelles (1985-95) de la production sédimentaire sont très variées, comme le montrent les études réalisées dans le bassin du Draix qui draine les terres noires de la nappe de Digne [32]. Si une ravine élémentaire (bassin de la Roubine de 0,133 ha) montre une forte ablation (110 à $190 \mathrm{t} / \mathrm{ha} / \mathrm{an}$ ), un bassin de plus grande taille fortement dénudé (bassin de Laval de 86 ha) montre des valeurs semblables (170 t/ha/an), alors qu'un bassin versant boisé (bassin du Bousquet, 108 ha) produit 220 fois moins $(0,50$ à $0,86 \mathrm{t} / \mathrm{ha} / \mathrm{an})$. Pour ces bassins versants élémentaires, les flux sont donc essentiellement fonction de l'état de la surface de terrain dénudée. À l'échelle de bassins versants kilométriques (entre 1 et $5000 \mathrm{~km}^{2}$ ), les valeurs de la production sédimentaire calculées à partir des remplissages lacustres alpins [3] sont comprises entre 10 à 150 $\mathrm{t} / \mathrm{km}^{2} / \mathrm{an}$, soit un taux de dénudation actuel ne dépassant pas des valeurs de $0,4 \mathrm{~mm} / \mathrm{an}$ (Fig. 8).

La production sédimentaire du bassin versant du lac du «Claps» est supérieure à toutes les valeurs actuelles calculées dans les Alpes (Fig. 8). Mais elle reste cependant inférieure à toutes les valeurs d'ablation glaciaire calculées dans les massifs alpins et jurassiens lors du dernier maximum glaciaire [25,33,34] La dénudation qui atteint $0,7 \mathrm{~mm} / \mathrm{an}$ au cours des 350 ans de fonctionnement du piège lacustre du «Claps » traduit certainement la dégradation hydro-climatique du Petit Age Glaciaire [13]. En effet dans les Alpes cette période correspond à une diminution de la température moyenne de 0,5 à $1{ }^{\circ} \mathrm{C}$, et probablement à une fréquence accrue d'été frais et humides, une augmentation des précipitations de forte intensité [35]. De plus dans la Drôme, comme sur l'ensemble du territoire français, la couverture forestière de l'époque moderne est très réduite et largement dégradée [36.37]

Le taux de production sédimentaire élevé enregistré par le lac du «Claps » de Luc-en-Diois durant 350 ans est lié à la nature lithologique de son bassin versant, mais aussi à un

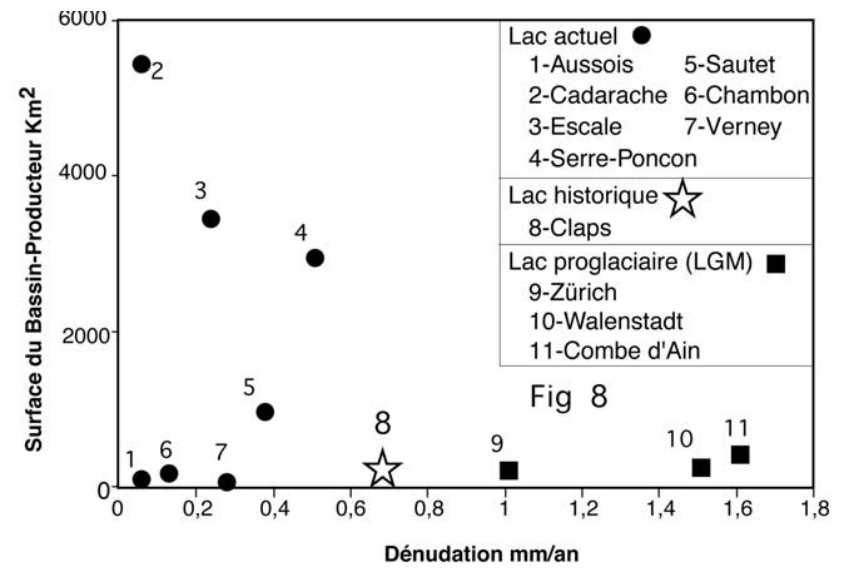

Fig. 8. Dénudation en fonction de la surface du bassin producteur calculé dans les Alpes pour des lac actuels (1 à 7 , d'après [3]); le lac historique du «Claps » (cette étude) et des lacs proglaciaires ayant fonctionnés durant le dernier maximum glaciaire $(9:$ [34] $1999 ; 10:[32]$ et $11:[33]$ Histogramm representing the slope in the watershed. Denudation rate versus surface of the drainage area basin calculate in the Alps for present day lakes (1 to 7, according to [3]); the historical lake of "Claps" (this study); and proglacial active during the Last Glacial Maximum (9: [34] $10:$ :32] and 11: [33]).

forçage hydro-climatique et anthropique. Ainsi les valeurs actuelles calculées pour la production sédimentaire ne sont que très peu représentatives du fait de leur faible intégration temporelle et ne peuvent donc pas être extrapolées de façon directe sur des périodes plus longues, séculaires à millénaires.

\section{Références}

[1] M. Hornung, F. Roda, S.J. Langan, A review of small catchment studies in western Europe, Producing hydrochemical budgets, Air Pollution Research, Report $\mathrm{n}^{\circ} 17$, Commission of European Communities, 1990, pp. 186.

[2] P. Ramez, M. Kellal, Production et tranfert de sédiments à l'échelle du bassin versant, C. R. Acad. Agri. Fr 80 (5) (1994) 15-23.

[3] L. Descroix, Les transports solides dans le Bez, Bull. Labo. Rhod. de Géomo. 27 (1991) 55-70.

[4] J.C. Olivery, J. Hoorelbeck, Erodabilité des terres noires de la vallée du Buëch, cahier ORSTOM, Pédologie vol XXV-12 (1990) 95-110.

[5] J.D. Milliman, R.H. Meade, World-delivery of river sediment to the oceans, Journal of Geology 91 (1983) 1-21.

[6] M. Meybeck, Les fleuves et le cycle géochimique des sédiments, thèse de l'Université de Paris VI, 1984, pp. 564.

[7] J.I. Svendsen, J. Mangerud, G.H. Miller, Denudation rates in the Arctic estimated from lake sediments on spitsberg, Svalbard, Paleogeo. Paleoclimat. Paleoecol. 76 (1989) 153-168.

[8] M. Campy, V. Bichet, Ch. Di-Giovanni, J. Richard, H. Richard, P. Olive, Evolution des flux de matière depuis 12000 ans dans la haute vallée du Doubs France, Bull. Soc. Géol. France 165 (1994) 383-402.

[9] J.J. Macaire, G. Bossuet, A. Choquier, C. Cocirta, P. De Luca, A. Dupis, I. Gay, E. Mathey, P. Guenet, Impact climatique et anthropique sur l'érosion mécanique en montagne cristalline de région tempérée pendant le Tardiglaciaire et l'Holocène. Analyse du bassin du lac de Chambon Puy-de-Dôme, France, C.R. de l'Académie des Sciences tome 320 série IIa 7 (1995) 579-585. 
[10] M. Parde, Le calcul des débits du Rhône et de ses affluents, Thèse Doctorat Université de Grenoble, 1925, pp. 168.

[11] N. Landon, H. Piegay, L'incision de deux affluents subméditerranéens du Rhône : la Drôme et l'Ardèche, Revue de Géographie de Lyon 69 (1) (1994) 63-72.

[12] M. Froment, Le «Claps » et l'ancien lac de Luc-en-Diois, in : A. Robert (Ed.), Luc-en-Diois, 1988, pp. 42.

[13] H. Lamb, Climate : Present past and future, Methuen, London, 1977, pp. 835.

[14] C. Pfister, The Little Ice Age, thermal and wetness indices for central Europe, Journal of interdisciplinary History 4 (1980) 145-172.

[15] J.P. Vernet, R. Horn, H. Badoux, G. Scolari, Etude structurale du Léman par sismique réflexion continue, Eclogae geol. Helv. 67 (3) (1974) 515-529.

[16] J.A. Hunter, S.E. Pullman, R.A. Burns, R.M. Gagne, R.L. Good, Shallow seismic reflection mapping the overburden-bedrock interface with the engineering seismograph- some simple techniques, Geophysics 49 (1984) 1381-1385.

[17] A. Pugin, S. Rossetti, Acquisition of land based high resolution seismic profils in glacial basins, two case studies in the Alpine foreland of Switzerland, Eclogae geol. Helv. 85 (2) (1992) 491-502.

[18] R.D. Miller, R.D. Markiewicz, C. Merey, J. Xia, G. Maples, Improvements in shallow high-resolution seismic reflection through PC-based systems, Computers \& Geosciences 21 (1995) 957-964.

[19] P. Van Rensbergen, M. de Batist, Ch. Beck, E. Chapron, Highresolution seismic stratigraphy of glacial to interglacial fill of a deep glacigenic lake : Lake Le Bourget, Northwestern Alps, France, Sedimentary geology 128 (1999) 99-129.

[20] W.M. Telford, L.P. Geldart, R.E. Sheriff, D.A. Keys, Prospection géophysique Tome 1 . Prospection sismique, La Barbannerie, Paris, 1984, pp. 240.

[21] R.W. Knapp, D.W. Steeples, Hight-resolution common-depth-point reflection profiling: field acquisition parameter design, Geophysics 51 (1986) 283-294.

[22] O. Yilmaz, Seismic data processing, investigation in geophysics, volume 2, Society of exploration in geophysicists, 1988, pp. 526.

[23] M. Lavergne, Méthodes sismiques, Technip, Paris, 1986, pp. 207.

[24] G. Matheron, Traité de géostatistique appliquée, tome 1 : théorie, Mémoire du BRGM, 14, 1962, pp. 338.

[25] J.F. Buoncristiani, Production sédimentaire détritique des systèmes glaciaires, Quantification des produits stockés dans un lac proglaciaire durant la dernière glaciation : exemple du lac de la Combe d'Ain (Jura, France), Thèse Université de Bourgogne, 1997, pp. 232.
[26] G.M. Brune, Trapp efficienty reservoir, Transaction Am. Geophysical Union 34 (1953) 407-418.

[27] H.G. Heinemann, Reservoir trap efficiency, in : R.F. Hadley, D.E. Walling (Eds.), Erosion and sediment yield, some methods of measurement, Geobooks, Nowich, 1984, pp. 201-218.

[28] V. Bichet, Impact des contraintes environnementales sur la production sédimentaire d'un bassin-versant Jurassien au cours du Postglaciaire, Le système limnologique de Chaillexon. Thèse Univ. de Bourgogne, 1997, pp. 206.

[29] G. Einsele, Sedimentary bassins :evolution, facies and sedimentary budget, Springer-Verlag, London, 1992, pp. 628.

[30] J.L. Probst, P. Amiotte Suchet, Fluvial suspended sediment transport and mechanical erosion in Maghreb (North Africa), Hydrological Sciences 37 (6) (1993) 621-637.

[31] W. Ying, E.R. Mei, Z. Dakuei, Sediment supply to the continental shelf by the major rivers of China, J. Geol. Soc. London 143 (1986) 935-944.

[32] D. Richard, N. Mathys, Historique, contexte technique et scientifique des BVRE de Draix. Caractéristiques, données disponibles et principaux résultats acquis au cours de dix ans de suivi, in : CEMAGREF (Ed.), Actes du colloque : les bassins versants expérimentaux de Draix, laboratoire d'étude de l'érosion en montagne, 1999, pp. 11-29.

[33] E. Schindler, Zur Geologie des Zürichees, Eclogae geol. Helv. 67 (1974) 163-196.

[34] B.U. Muller, Paraglacial sedimentation and denudation processes in an Alpine valley of Switzerland. An approche to the quantification of sediment budgets, Geodinamica Acta 12 (5) (1999) 291-301.

[35] C. Pfister, Klimageschichte der Schweiz 1525-1860, Das Klimat der Schweiz von 1525-1860 und seine Beduntung in der Gesschischte von Bevölgerung und Landwirtschaft, Verlag Paul Haupt, Bern, 1988.

[36] M. Coûteaux, Caractérisation pollenanalytique en Oisans des milieux forestiers et des milieux supraforestiers dans l'actuel et le passé, Actes du colloque de Perpinya (1981) 139-159.

[37] T. Nakagawa, Etude palynologique dans les Alpes Françaises, Centrales et Méridionales : histoire de la végétation Tardiglaciaire et Holocène, thèse de l’Université de Marseille, 1998, pp. 211. 